\title{
Morfometría geométrica en elementos óseos postcraneales de los caballos del Pleistoceno tardío en México: implicaciones taxonómicas y ecomorfológicas
}

\author{
Alejandro H. Marín-Leyva ${ }^{1,3^{*}}$, María Teresa Alberdi ${ }^{2}$, María Luisa García-Zepeda ${ }^{3}$, \\ Javier Ponce-Saavedra' ${ }^{4}$, Peter Schaaf ${ }^{1}$, Joaquín Arroyo-Cabrales ${ }^{5}$ y Markus Bastir ${ }^{2}$
}

\author{
${ }^{1}$ Laboratorio Universitario de Geoquímica Isotópica (LUGIS), Instituto de Geofísica, Universidad Nacional Autónoma de México, \\ Circuito de la Investigación Científica S/N, Ciudad Universitaria, Del. Coyoacán, 04150, Ciudad de México, México. \\ ${ }^{2}$ Departamento de Paleobiología, Museo Nacional de Ciencias Naturales, CSIC, José Gutiérrez Abascal, 2, 28006 Madrid, España. \\ ${ }^{3}$ Laboratorio de Paleontología, Facultad de Biología, Universidad Michoacana de San Nicolás de Hidalgo, \\ Edif. R $2^{\circ}$ Piso, Ciudad Universitaria, C.P. 58030, Morelia, Michoacán, México. \\ ${ }^{4}$ Laboratorio de Entomología "Biol. Sócrates Cisneros Paz", Facultad de Biología, Universidad Michoacana de San Nicolás de Hidalgo, \\ Edif. B4 2 Piso, Ciudad Universitaria, C.P. 58030, Morelia, Michoacán, México. \\ ${ }^{5}$ Laboratorio de Arqueozoología "M. en C. Ticul Álvarez Solórzano", Subdirección de laboratorio y Apoyo Académico, \\ Instituto Nacional de Antropología e Historia, Moneda \#16, Col. Centro, 06060, Ciudad de México, México. \\ *alexmarin@igeofisica.unam.mx, alexmanleyva@gmail.com
}

\section{RESUMEN}

El género Equus es el último representante de la Familia Equidae cuya historia evolutiva tiene aproximadamente 55 millones de años. Este género es abundante en los depósitos mexicanos del Pleistoceno. En el presente trabajo se analizan elementos de las extremidades distales (metacarpos y metatarsos terceros, primeras y segundas falanges del tercer dígito) de Equus cedralensis, E. conversidens y E. mexicanus de tres localidades del Rancholabreano (0.21-0.16 a 0.095 Ma) en México mediante técnicas de morfometría geométrica. El objetivo es conocer si la forma de cada elemento estudiado difiere entre las especies de équidos, además de encontrar alguna relación ecomorfológica (forma vs. sustrato). Los resultados comprueban la existencia de tres grupos diferentes de forma que se relacionan con las tres especies de caballos registradas en las localidades estudiadas, además de existir elementos diagnósticos explicativos y poco explicativos para la diferenciación de las tres especies de caballo del Pleistoceno tardío en México. Con base en los tres diversos morfotipos se deduce que las especies pudieron vivir en microhábitats diferentes y por lo tanto coexistir en el mismo macrohábitat. Finalmente, la variedad de morfotipos en las localidades en estudio permite inferir que eran ambientes heterogéneos.

Palabras clave: Rancholabreano; extremidades distales; équidos; morfometría geométrica; México.

\author{
ABSTRACT \\ The genus Equus is the last member of the Equidae family whose \\ evolutionary history is about 55 million years. This horse is abundant \\ in Mexican Pleistocene deposits. Elements of the distal extremities (third
}

metacarpals and metatarsals, first and second phalanx of the third digit) of three horse species (Equus cedralensis, E. conversidens and E. mexicanus) from three localities of Mexican Rancholabrean (0.21-0.16 a $0.095 \mathrm{Ma}$ ) were analyzed using geometric morphometric techniques. The aim is know whether the shape of each element studied differs between species of horses and also find some ecomorphological connection (form vs. substrate). The results prove the existence of three different groups related with the three species of horse registered in the localities studied; also, the existence of diagnostic elements for the differentiation of the three species of the late Pleistocene horse in Mexico. Based on the three diverse morphotypes is deduced that the species could live in different microhabitats and therefore be able to coexist in the same macrohabitat. Finally, the diversity of morphotypes suggests heterogeneous environments in the study localities.

Key words: Rancholabrean; distal extremities; equids; geometric morphometric; Mexico.

\section{INTRODUCCIÓN}

La familia Equidae Gray, 1821 es un grupo monofilético cuyo registro más antiguo proviene del Eoceno temprano de Norteamérica. Los miembros de esta familia tuvieron una amplia radiación filogenética y adaptativa en el Mioceno medio aproximadamente hace 13-9 Ma. Ésta fue evidente sobre todo en el número de taxones (11-13 géneros), en sus elementos dentales (hipsodontia), en sus extremidades (reducción en el número de dedos funcionales) y en la masa corporal (aumento del tamaño) (Alberdi et al., 2014; MacFadden, 1992).

Los équidos fueron disminuyendo en diversidad de géneros progresivamente a partir del Mioceno tardío hace aproximadamente $9 \mathrm{Ma}$.

Marín-Leyva, A.H., Alberdi, M.T., García-Zepeda, M.L., Ponce-Saavedra, J., Schaaf, P., Arroyo-Cabrales, J., Bastir, M., 2019, Morfometría geométrica en elementos óseos postcraneales de los caballos del Pleistoceno tardío en México: implicaciones taxonómicas y ecomorfológicas: Revista Mexicana de Ciencias Geológicas, v. 36, núm. 2, p. 195-206. 
Durante el Pleistoceno tardío existieron posiblemente dos géneros de equinos: Haringtonhippus un género descrito recientemente para los denominados "New World Stilt-legged" (Heintzman et al., 2017) y Equus ampliamente distribuido con al menos dos o tres especies simpátricas. Finalmente, hace aproximadamente $0.01 \mathrm{Ma}$ se extinguieron con otros grandes mamíferos en Norteamérica (Heintzman et al., 2017; MacFadden, 1992; Winans, 1985, 1989).

Actualmente existen sólo siete especies de la familia Equidae, las cuales pertenecen al género Equus. Incluyen los caballos domésticos y salvajes, los asnos africanos y asiáticos así como las cebras: Equus ferus, E. africanus, E. hemionus, E. kiang, E. grevyi, E. quagga, E. zebra. Estos taxones se encuentran distribuidos en Eurasia y África, habitando principalmente meso-pastizales a sabana semiárida, tierras bajas áridas, montañas y desiertos (Rubenstein, 2011).

La conducta ecológica es un factor importante para determinar el nicho de una especie, por lo tanto, en parte define el rango de presiones de selección a los que los animales están sujetos (Van Asperen, 2010). MacFadden (1992) menciona que existe evidencia de cómo el comportamiento ecológico y de organización social de los caballos pacedores del Mioceno medio es similar al de los équidos modernos. El desarrollo de ambientes extensos de pastizal con una calidad de hábitat pobre y fuertes variaciones estacionales, el aumento del tamaño corporal y de territorio, la necesidad de evadir depredadores a través de la carrera, los llevó a evolucionar hacia un estilo de vida con mayor movilidad. Por lo tanto, la gama de adaptaciones de dieta y locomoción que pueden ser observadas en los équidos modernos encuentra su origen en sus ancestros neógenos (Van Asperen, 2010).

Desde un punto de vista funcional, el aspecto principal de la movilidad que influye en la proporción y morfología de los huesos de las extremidades es la velocidad máxima de carrera y la resistencia (Gregory, 1912; Steudel y Beattie, 1993).

En caballos, así como en otras especies, la anatomía de las extremidades no puede ser optimizada para máxima velocidad de carrera sino para reducir al mínimo el consumo de energía durante todas las formas de locomoción (Christiansen, 2002). En mamíferos corredores como los caballos, la longitud total de la extremidad y la longitud relativa de los segmentos de la extremidad distal se correlacionan positivamente con la máxima velocidad de carrera (Garland y Janis, 1993; Van Valkenburgh, 1987).

En caballos se cree que los segmentos distales de las extremidades: radio (R), tibia (T), metacarpo (MC) y metatarso (MT) son ligeramente más largos en especies como: E. hemionus, E. kiang, E. ferus que se relacionan con ambientes abiertos, mientras que los équidos actuales como E. quagga y E. zebra tienen estos segmentos ligeramente más cortos y se relacionan con ambientes cerrados y/o de montaña. La anchura de las terceras falanges, así como la superficie articular de la segunda falange en los équidos, se relacionan con el tipo de sustrato: lodo, arena o nieve; por ejemplo: en E. zebra son angostas por vivir en suelos duros de montaña; E. kiang y E. ferus las tienen anchas por habitar suelos blandos (arena y nieve); mientras que en E. hemionus, E. africanus y E. quagga son intermedias debido a que se mueven en suelos duros no montañosos. La robustez o gracilidad de los metápodos ( $\mathrm{MC}$ y MT) podría estar vinculada con condiciones secas o húmedas, como en el caso de E. ferus que es robusto y habita zonas relativamente más húmedas en comparación con $E$. hemionus que es grácil y vive en zonas más secas (Eisenmann, 1984).

La taxonomía del género Equus, en general, es complicada sobre todo para los depósitos del Pleistoceno. En México se han registrado hasta 25 especies, con 13 nombres de localidades tipo mexicanas, descritas a partir de caracteres morfológicos dentales altamente variables por ser hipsodontos, en su mayoría hoy en día no son válidos (Alberdi et al., 2014; Winans, 1985, 1989). Hoy en día se consideran válidas cinco especies encontradas en el Rancholabreano (0.21-0.16 a 0.095 Ma sensu Bell et al., 2004) NALMA (North American Land Mammal Age, Edad de Mamíferos Terrestres de Norteamérica): Equus cedralensis, E. conversidens, E. mexicanus, E. excelsus y E. francisci, algunos de estos taxones tienen una mayor distribución geográfica que otras, posiblemente por una preferencia de dieta o de tipo hábitat (Alberdi et al., 2014; Priego-Vargas et al., 2016). Actualmente, el estudio de los restos fósiles de équidos en México se encuentra en desarrollo, y análisis más profundos (filogenéticos, biogeográficos, ecológicos y evolutivos) se deben realizar con el fin de entender mejor la familia Equidae (Priego-Vargas et al., 2016).

Las especies más estudiadas con datos morfométricos lineales en elementos craneales y postcraneales para el Rancholabreano son: Equus cedralensis Alberdi et al., 2014 un équido de talla pequeña, de extremidades gráciles y baja masa corporal, que pudo haber habitado zonas con suelos compactados, con una alimentación mediana a altamente abrasiva y plantas $\mathrm{C}_{4}$; E. conversidens Owen, 1869 un caballo de talla media, extremidades robustas y mediana masa corporal, que pudo habitar zonas tanto de suelos compactos como sueltos, con una dieta compuesta de alimentos altamente abrasivos y plantas $\mathrm{C}_{4}$; y E. mexicanus Hibbard, 1955, especie de talla grande, extremidades robustas y gran masa corporal que pudo haber vivido en áreas de suelos poco compactados o sueltos, con una preferencia alimenticia por recursos mediana a altamente abrasivos y plantas $\mathrm{C}_{3} \mathrm{y} \mathrm{C}_{4}$ (Alberdi et al., 2014; Barrón-Ortiz et al., 2014; Bravo-Cuevas et al., 2011; Eisenmann, 1984; Marín-Leyva, 2011; Marín-Leyva, et al. 2016a, b; Pérez-Crespo et al., 2009).

Todas las características deducidas a partir de las distintas morfologías de los restos de extremidades de estas especies se hicieron en relación con parientes actuales (Alberdi et al., 2014; Eisenmann, 1984; Marín-Leyva, 2011) mientras que el tipo de alimentación se infirió a partir de análisis de meso y microdesgaste e isótopos estables de carbono y de oxígeno (Barrón-Ortiz et al., 2014; Bravo-Cuevas et al., 2011; Marín-Leyva et al., 2016a, b; Pérez-Crespo et al., 2009).

La morfometría es básicamente el análisis cuantitativo de la forma. El estudio de la forma implica una aproximación a las causas de variación y transformación morfológica de esta misma, atribuida a una variedad de procesos biológicos que producen las diferencias en la forma entre individuos o sus partes, como el desarrollo ontogénico, la adaptación a los factores geográficos locales, o diversificación evolutiva a largo plazo (Slice, 2007; Zelditch et al., 2004).

La morfometría tradicional o lineal se encarga del estudio de medidas como, por ejemplo, la longitud, profundidad y anchura entre dos puntos de una forma (Fink, 1990; Hammer y Harper, 2006; Zelditch et al., 2004). Estos trabajos en caballos del Pleistoceno están enfocados principalmente en su taxonomía con aproximaciones paleoecológicas (Alberdi et al., 1998, 2003, 2014; Eisenmann, 1984; Prado y Alberdi, 1994; Winans, 1985, 1989).

Hay algunos trabajos de morfometría geométrica (MG) en caballos del Pleistoceno que tienen un enfoque ecomorfológico (Bignon et al., 2005; Van Asperen, 2010) sobre cómo las especies se adaptan a cambios ambientales a través de un rango de espacio temporal y cuanta variación puede ocurrir en una especie en respuesta a la presión ambiental (Van Asperen, 2010). También existe un ejemplo que utiliza la MG con fines taxonómicos en équidos del Mioceno (Calvo y Salesa, 2006).

En el presente trabajo se analizaron elementos postcraneales de tres especies de caballos (Equus cedralensis, E. conversidens y E. mexicanus) de tres localidades del Pleistoceno tardío, dos del centro occidente y una del centro norte de México, mediante técnicas de morfometría geométrica en dos dimensiones, con la finalidad de conocer si la forma de cada elemento estudiado difiere entre especies, además de determinar si estas disimilitudes tienen aplicaciones taxonómicas y ecomorfológicas (forma vs. sustrato). 


\section{DESCRIPCIÓN DE ÁREAS DE ESTUDIO}

De las tres áreas de donde proviene el material en estudio dos proceden de la provincia morfotectónica del Cinturón Volcánico Trans-Mexicano (CVTM) y una está ubicada en la Sierra Madre Oriental (SMOr). Las tres tienen una edad del Pleistoceno tardío (sensu Ferrusquía-Villafranca et al., 2010). El área de La Cinta-Portalitos (LC-PT) está localizada entre las comunidades de La Cinta municipio de Cuitzeo, Michoacán, y Portalitos municipio de Uriangato, Guanajuato, en la Cuenca de Cuitzeo (Figura 1). El área de estudio de La Piedad-Santa Ana (LP-SA) se localiza en la cuenca del bajo Lerma entre las ciudades de La Piedad de Cabadas municipio de La Piedad, Michoacán, y Santa Ana Pacueco municipio de Pénjamo, Guanajuato (Figura 1). La localidad de Cedral (CE) municipio de Cedral en San Luis Potosí se encuentra en la cuenca del Altiplano. Los restos fósiles de vertebrados de estas localidades se asignan al Rancholabreano. El ensamble paleontológico es variado y extenso en las tres localidades, comprendiendo numerosos taxones que incluyen mamíferos, reptiles y anfibios. En los sitios de estudio los perisodáctilos son el componente más numeroso en relación a cantidad de elementos óseos encontrados, seguido por los proboscidios, los cetartiodáctilos y los roedores.

El área de la LC-PT (Figura 1) tiene una geomorfología heterogénea que se subdivide en cuatro zonas: NE, con volcanes tipo escudo y fallas normales, parte Norte del graben de Cuitzeo; NW, edificios volcánicos monogenéticos y tipo escudo; SW, presencia de las rocas volcánicas más antiguas y $\mathrm{S}$, lago actual y zonas con drenaje alterado por la activad agrícola (Marín-Leyva, 2011; Marín-Leyva et al., 2016a, 2016b). Seis facies litológicas y paleontológicas se reconocen para esta área: Facies I, lacustre, arcillas y diatomita, baja energía y lago estable; Facies II, ígnea, arenas volcánicas, energía media; Facies III, fluviolacustre, arena y arcilla, energía media; Biofacies IV, fluvio-lacustre, microconglomerado, energía media con restos fósiles de macro y micro-vertebrados; Facies V, lacustre, arcillas y diatomita, baja energía; y Facies VI, suelos, limo, baja energía (Marín-Leyva, 2011; Marín-Leyva et al., 2016a, 2016b). La fauna asociada en el área incluye xenartros (Nothrotheriidae), carnívoros (Canidae), roedores (Cricetidae y Hydrochoeridae), perisodáctilos (Equidae), cetartiodáctilos (Bovidae, Camelidae, Cervidae y Tayassuidae) y proboscidios (Elephantidae). Otros grupos de vertebrados son los anuros, caudados, quelonios y escuamatos (Cervantes-Barriga, 2015, 2018; Díaz-Sibaja, et al., 2014, 2018; Eng-Ponce, 2018; García-Zepeda, 2006; Gutiérrez-Bedolla et al., 2016; Marín-Leyva et al., 2016a, 2016b; Moreno-Flores, 2018; PérezGonzáles y Godínez-García, 2007; Plata-Ramírez, 2012).

El área LP-SA (Figura 1) tiene una geomorfología homogénea, subdividida en cuatro zonas: NW, parte del río Lerma entre sierras y colinas; W, parte de las planicies de Chapala; SW, edificio volcánico del Cerro Grande; SE, parte de las grandes planicies del río Lerma (Marín-Leyva, 2011; Marín-Leyva et al., 2016a, 2016b). Para esta área se reconocen seis facies litológicas y paleontológicas: Facies I, fluviolacustre, arcilla con arena, energía media y alta; Facies II, volcánica, arena, energía media; Biofacies III, micro y macro-conglomerados, alta energía, con fósiles de macrovertebrados; Facies IV, arena y limo, energía media y alta; Facies V, lacustre, arcilla y diatomea, energía baja; y Facies VI, suelos, arcilla y limo, energía baja (Marín-Leyva,

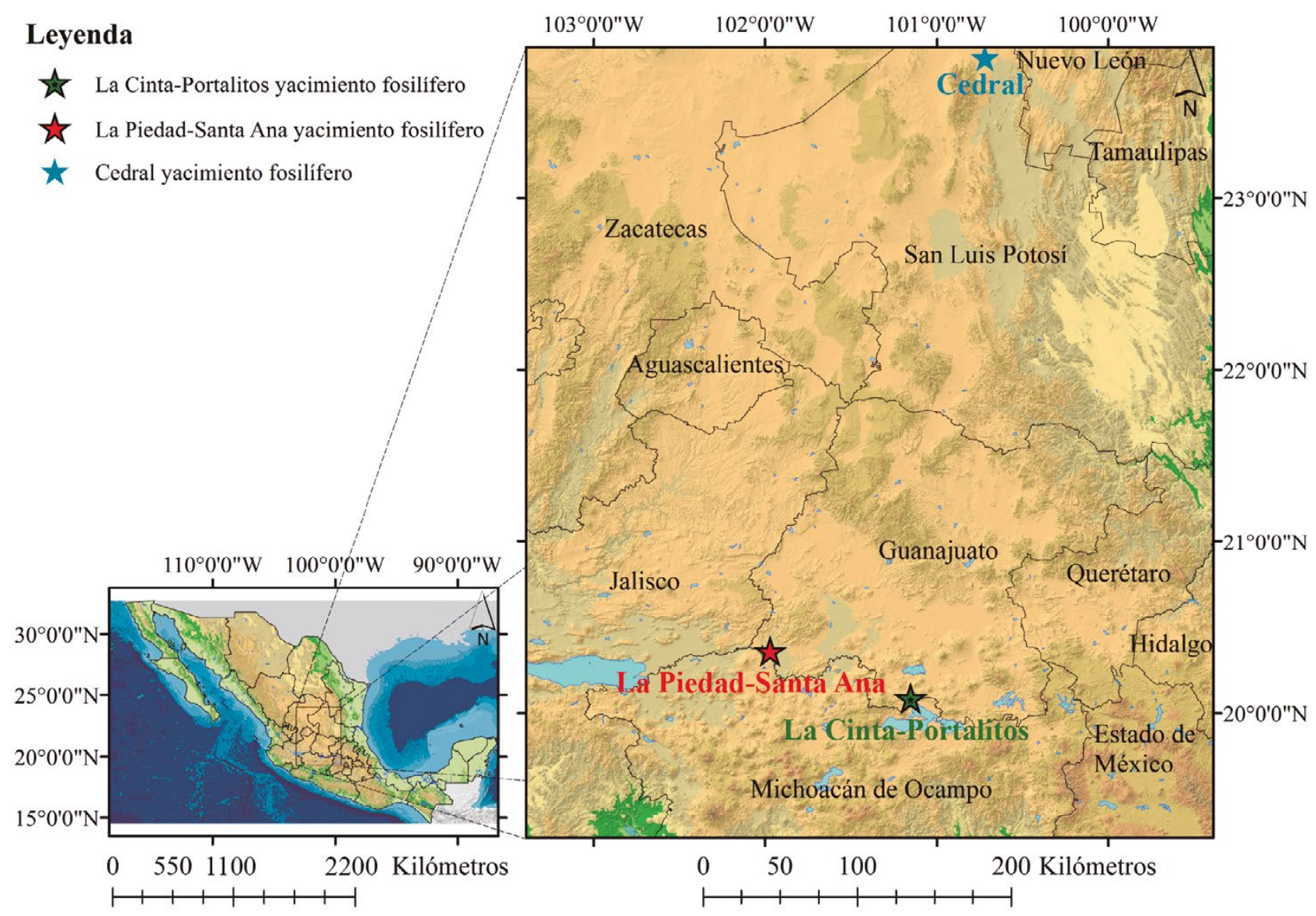

Figura 1. Localización geográfica de La Cinta-Portalitos, La Piedad-Santa Ana y Cedral, yacimientos fosilíferos en estudio con restos de équidos del Pleistoceno tardío en México. 
2011; Marín-Leyva et al., 2016a, 2016b). En esta área también se tiene la presencia principalmente de carnívoros (Canidae), perisodáctilos (Equidae), cetartiodáctilos (Bovidae, Camelidae) y proboscidios (Elephantidae) (Cervantes-Barriga, 2015; Díaz-Sibaja et al., 2018; Gutiérrez-Bedolla et al., 2016; Marín-Leyva et al., 2016a, 2016b; PlataRamírez, 2012).

El área del CE (Figura 1) presenta una geomorfología homogénea que se caracteriza por una llanura plana, que forma parte de un valle flanqueado por tres sierras, la de catorce al SE, la de Jicote SW, y la sierra El Azul W (Reyes y Flores, 2012). Para esta área se reconocen al menos 16 niveles estratigráficos agrupados en nueve facies litológicas y paleontológicas: Facies I (XVI, XV y XIV), lagunar, arcilla, energía baja; Facies II (XIII), lagunar-pantano, arcilla, baja energía; Biofacies III (XII), pantano, arcilla, baja energía, con materia orgánica humificada y restos fósiles de macrovertebrados; Biofacies IV (XI, X y IX), lagunarpantano, limo y arcilla, energía baja, restos fósiles de invertebrados y macrovertebrados; Biofacies V (VIII), acarreo, arcilla, energía baja a media, palizada, con abundantes troncos, restos de macrovertebrados defensa y huesos; Facies VI (VII y VI), lagunar, arcilla, energía baja, con restos de moluscos; Biofacies VII (V y IV), evaporítico-hidrotermal, limo y arcilla, baja energía, con restos fósiles (defensa de mamut); Facies VIII (III), evaporítico y de pantano, arcilla, baja energía; Facies IX (II y I), pantano, arcilla, limo arena, baja a media energía (Flores-Díaz, 2012). Varios horizontes de fauna se encuentran en el depósito, estas capas tenían abundantes restos de huesos, mayormente aislados y dispersados, sin embargo, una gran concentración de huesos de caballos fue descubierta en las Biofacies III y IV entre las capas X y XII con fechamientos de radiocarbono entre 30,000 y 25,000 años AP (antes del presente) (Alberdi et al., 2014; Mirambell, 2012). En esta área se tiene registro de carnívoros (Canidae y Felidae), perissodactilos (Equidae y Tapiridae), cetartiodáctilos (Camelidae) y proboscidios (Elephantidae y Mammutidae), así como micromamíferos y otros vertebrados (Álvarez S. y Polaco, 1982).

Una composición similar de fauna se encuentra tanto en los sitios del centro occidente (LC-PT y LP-SA) como en el de centro norte (CE) de México. Sin embargo, en algunos sitios tienen especies exclusivas y una mayor riqueza de restos fósiles.

Para el área donde se localiza LC-PT (lacustre) existen diferentes estudios paleoambientales que incluyen análisis de diatomeas y polen, esto último indicando la presencia arbustos y herbáceas (Cheno-Am, Ambrosia spp., Asteraceae indet., Cirsium spp., and Thalictrum spp.). (Caballero et al., 2010; Israde-Alcántara et al., 2002, 2010). En el área de LP-SA (fluvial) no existe ningún trabajo paleoambiental que utilice análisis de microfósiles.

En el área del centro norte de México CE (fluvial y lacustre) se han hecho diferentes trabajos paleoambientales a partir de microvertebrados, moluscos y polen, que señalan la presencia de árboles, herbáceas y pastos (Cupressus, Juniperus, Taxodium, Pinus, Quercus, Poaceae y Asteraceae), así como cambio de ambiente frío y húmedo hacia templado y árido (Olivera-Carrasco, 2012; Pérez-Crespo et al., 2009; Sánchez y Alvarado, 2012).

\section{MATERIALES Y MÉTODOS}

Los fósiles se encuentran depositados en la Colección Paleontológica de la Universidad Michoacana de San Nicolás de Hidalgo, Facultad de Biología, UMSNH, Morelia, Michoacán; en la Colección Paleontológica de la Organización Especial de Investigación, Museo de Sitio de La Piedad Cabadas, Michoacán y la Colección Paleontológica (DP-INAH) del Laboratorio de Arqueozoología "M. en C. Ticul Álvarez Solórzano" Subdirección de Laboratorios y Apoyo Académico, Instituto Nacional de Antropología e Historia, Ciudad de México.

La morfometría geométrica (MG) es el estudio de las coordenadas cartesianas de los puntos anatómicos (landmarks o semi-landmarks) que deben cumplir ciertas características como: ser homólogos, consistentes y que cubran adecuadamente la forma a estudiar. La MG se apoya en técnicas estadísticas multivariantes como son el análisis de procrustes generalizado (APG) el cual coloca los landmarks de todos los especímenes en un sistema común coordinado, en donde las diferencias en la configuración de los landmarks reflejen las disimilitudes en la estructura de las formas. El análisis de componentes principales (ACP), el análisis de varianza canónica (AVC) y el análisis de funciones discriminantes (AD) se utilizan para explorar y clasificar grupos de formas (Slice, 2007; Zelditch et al., 2004).

Para los análisis de morfometría geométrica se usaron los métodos y técnicas propuestos por Bookstein (1991), Zelditch et al. (2004) y Slice (2007), utilizando los siguientes programas: TPSutilW32, TPSdig2W32 y MorphoJ 1.04a.

Se analizaron restos de las tres especies de caballos, donde se incluyen las localidades de Michoacán (LC-PT, LP-SA y EC). Los restos que se seleccionaron, dependiendo de su estado de conservación y ontogenia, fueron 16 metacarpos (MCIII) (LP-SA: E. cedralensis (3) y E. conversidens (1); CE: E. cedralensis (5), E. conversidens (2) y E. mexicanus (5)), 17 metatarsos (MTIII) (LC-PT: E. cedralensis (1); LP-SA: E. conversidens (1) y E. mexicanus (1); CE: E. cedralensis (6) y E. mexicanus (8)), 54 primeras falanges (1FIII) (LC-PT: E. cedralensis (2) y E. conversidens (9); LP-SA: E. conversidens (2) y E. mexicanus (1); CE: E. cedralensis (21), E. conversidens (4) y E. mexicanus (15)) y 56 segundas falanges (2FIII) (LC-PT: E. cedralensis (4) y E. conversidens (16); LP-SA: E. cedralensis (1), E. conversidens (1); CE: E. cedralensis (19), E. conversidens (2) y E. mexicanus (13)) (Figura 2; para mayor información, número de colección y yacimiento de origen, ver el Suplemento Electrónico). Debido al estado de conservación de las terceras falanges del tercer dedo no fue posible realizar análisis de MG en estos elementos, sin embargo, utilizamos los 1FIII y 2 FIII para hacer inferencias de la morfología de la 3FIII.

A cada uno de los elementos seleccionados se les tomó una fotografía con la misma escala, la misma distancia entre el ocular y el fósil, el mismo ángulo y la misma máquina fotográfica (Nikon D5200).

Para el análisis de las fotografías se hizo el siguiente procedimiento y se utilizaron los siguientes softwares: el programa TPSutilW32 para crear un archivo TPS, después el mismo archivo se abrió e indicó la escala con el TPSdig2W32, en el cual se colocaron los Landmarks (14 en MCIII, 17 en MTIII, 10 en 1FIII, 8 en 2FIII), los cuales fueron señalados en puntos homólogos y se eligieron con base en las variables de morfometría lineal (Eisenmann et al., 1988) (Figuras 3a, 3b, 4a y 4b). El siguiente paso, usando el software MorphoJ 1.04a, consistió en hacer un análisis general de procrustes (AGP), con el cual se generó una matriz de covarianza, que se utilizó para hacer un análisis de componentes principales con la finalidad de explorar la variación de forma de los distintos elementos (un ACP por cada uno) y el ordenamiento de formas.

Finalmente, mediante MorphoJ 1.04a, usando las coordenadas procrustes se hizo un análisis de varianza canónica (AVC) para cada uno de los elementos. Este método encuentra un conjunto de ejes (combinación lineal de variables) que permite discriminar entre dos o más grupos; para esto se usó la clasificación original de cada uno de los huesos analizados en Alberdi et al. (2014) y Marín-Leyva (2011), los cuales están organizados en tres grupos: E. mexicanus, E. conversidens y E. cedralensis. Todo ello, con la finalidad de corroborar o no la correcta clasificación que se hizo con variables morfométricas lineales y conocer si a través del AVC, realizado con los datos o variables de MG (en este caso, rasgos de la forma), explican la separación de grupos. El 

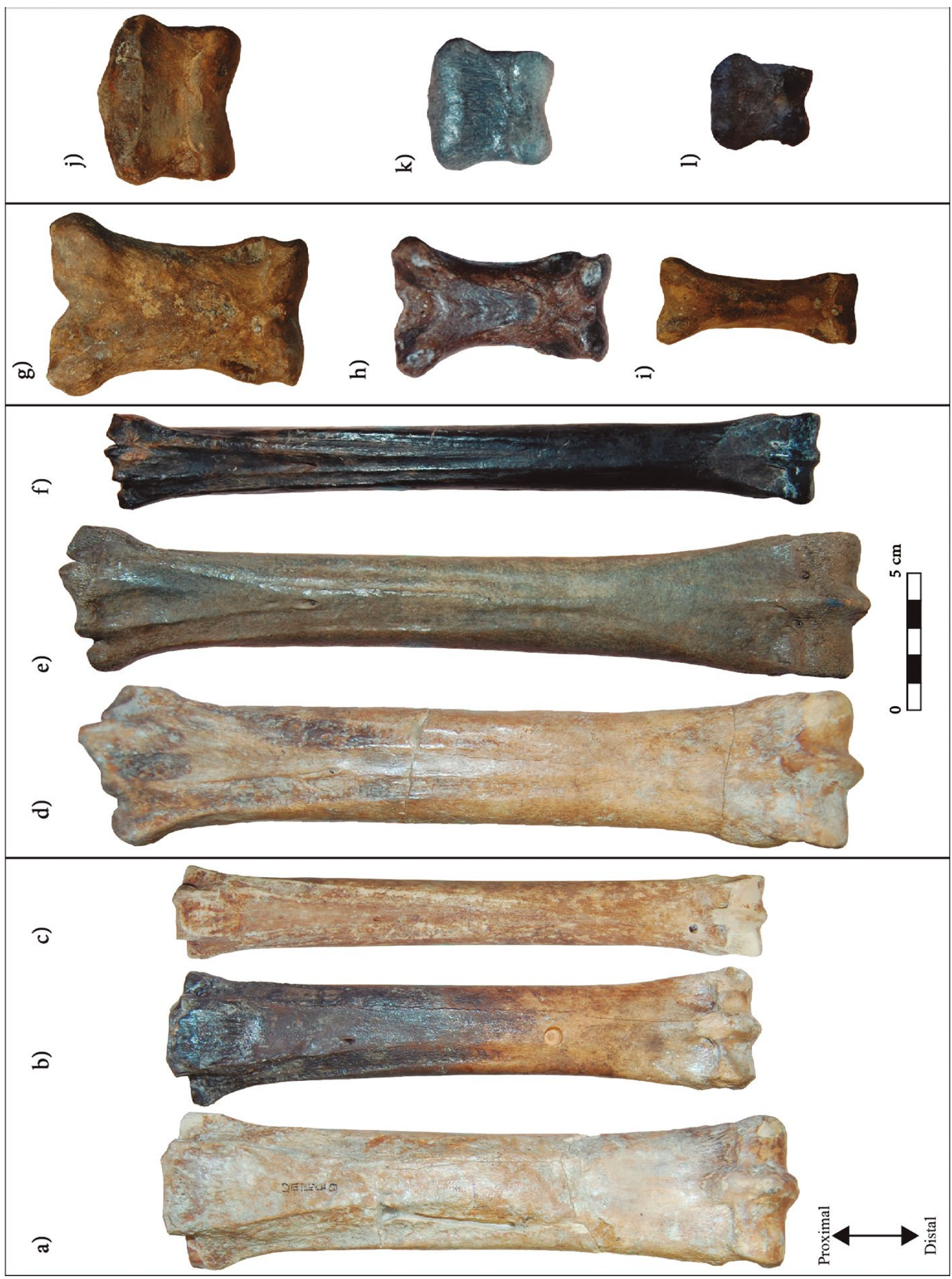

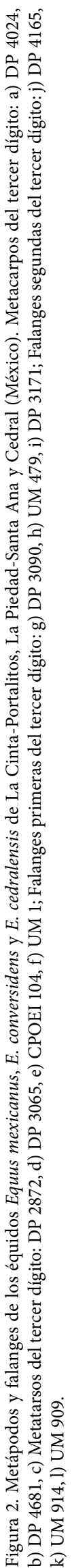


AVC tiene una prueba de significancia entre los grupos y calcula las variables canónicas de todos los especímenes.

\section{RESULTADOS}

A continuación, se exponen los resultados obtenidos de ACP (Tabla 1, Figuras 5a, 5b, 6a y 6b), y de AVC (Tabla 2, Figuras 7a, 7b, 8a y 8b).

En el ACP del MCIII los dos primeros componentes explican el $90.77 \%$ de la varianza. El componente 1 reúne el $85.55 \%$ y el 2 el $5.21 \%$ de la varianza (Tabla 1). Con el primer componente se lograron separar dos grupos: una forma grácil y alargada, la mayoría asignados a la especie E. cedralensis, y una de forma robusta, la mayoría pertenecientes a la especie E. mexicanus; y entre ambos una forma intermedia donde se encuentran individuos de E. conversidens y de E. cedralensis (Figura 5a).
En el ACP del MTIII los dos primeros componentes explican el $74.56 \%$ de la varianza. El componente 1 reúne el $55.49 \%$ y el 2 el $19.07 \%$ de la varianza (Tabla 1). Con el primer componente se lograron separar dos grupos, una forma grácil y alargada la mayoría asignados a la especie E. cedralensis, y uno de talla robusta la mayoría pertenecientes a la especie E. mexicanus, y entre ambos una forma intermedia donde se encuentra el único individuo de E. conversidens así como otros de E. cedralensis y E. mexicanus (Figura 5b).

En el ACP de la 1FIII los dos primeros componentes explican el $89.18 \%$ de la varianza. El componente 1 reúne el $84.70 \%$ y el 2 el $4.49 \%$ de la varianza (Tabla 1). Con el primer componente se lograron separar tres grupos, una forma grácil y alargada en la parte de la diáfisis y angosta en las articulaciones proximales y distales, la mayoría asignados a la especie E. cedralensis, y una forma no muy grácil o robusta compuesta en su mayoría por individuos de E. conversidens y algunos de E. cedralensis y E. mexicanus, y un grupo de forma robusta a)

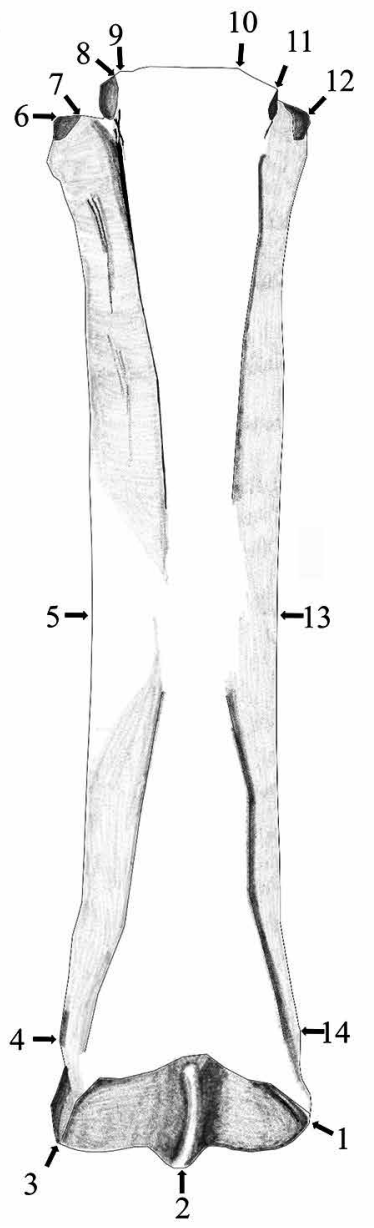

b)

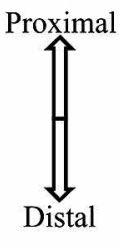

Figura 3. Landmarks de los Metacarpos y Metatarso terceros. a) Metacarpo: 1. Borde del cóndilo lateral, 2. Borde de la quilla, 3. Borde del cóndilo medial, 4. Ancho máximo medial de superficie supra-articular distal, 5. Ancho mínimo medial de la diáfisis, 6. Borde superior externo de la superficie articular del II metacarpo, 7. Borde superior interno de la superficie articular del II metacarpo, 8. Borde externo palmar de la superficie articular del trapezoide, 9. Borde interno palmar de la superficie articular del trapezoide, 10. Borde interno palmar de la superficie articular del uncinato, 11. Borde externo palmar de la superficie articular del uncinato, 12. Borde de la superficie articular del IV metacarpo, 13. Ancho mínimo lateral de la diáfisis, 14. Ancho máximo lateral de superficie supra-articular distal. b) Metatarso: 1. Borde del cóndilo lateral, 2. Borde de la quilla, 3. Borde del cóndilo medial, 4. Ancho máximo medial de superficie supra-articular distal, 5. Ancho mínimo medial de la diáfisis, 6. Borde inferior externo de la superficie articular externa del II metatarso, 7. Borde superior externo de la superficie articular externa del II metatarso, 8. Borde superior interno de la superficie articular externa del II metatarso, 9. Borde inferior de la superficie articular interna del II metatarso, 10. Borde superior de la superficie articular interna del II metatarso, 11. Borde superior de la superficie articular interna del IV metatarso, 12. Borde inferior de la superficie articular interna del IV metatarso, 13. Borde superior interno de la superficie articular externa del IV metatarso, 14. Borde superior externo de la superficie articular externa del IV metatarso, 15. Inicio de la curvatura en la diáfisis lateral en la zona proximal, 16. Ancho mínimo lateral de la diáfisis, 17. Ancho máximo lateral de superficie supra-articular distal. 
a)

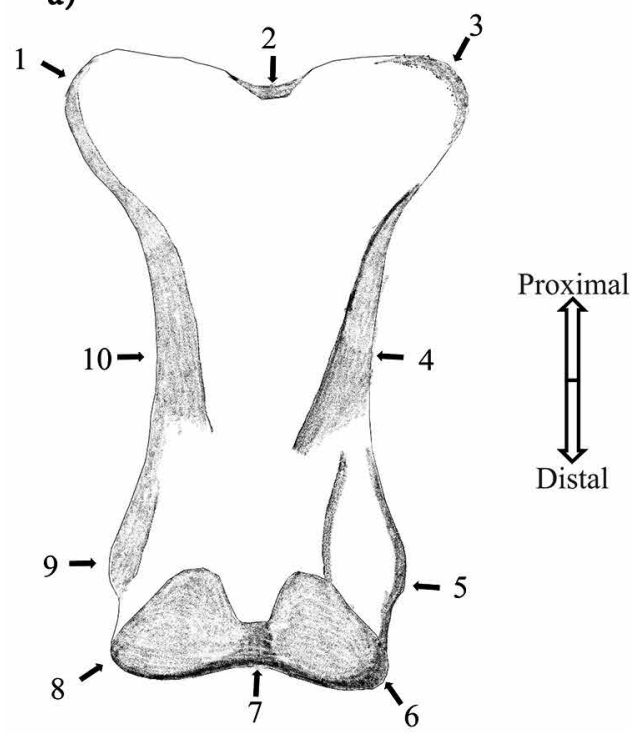

b)

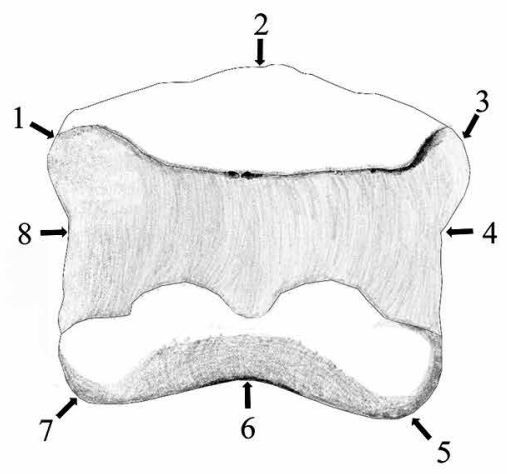

Figura 4. Landmarks de la primera y segunda falange del tercer dígito. a) Primera falange del tercer dígito: 1. Borde del tubérculo de inserción lateral, 2. Borde palmar del estrechamiento intermedio, 3. Borde del tubérculo de inserción medial, 4. Ancho mínimo medial de la diáfisis, 5 . Borde del tubérculo de inserción ligamentosa medial, 6. Borde del cóndilo medial, 7. Borde medio de la superficie articular proximal, 8. Borde del cóndilo lateral, 9. Borde del tubérculo de inserción ligamentosa medial, 10. Ancho mínimo lateral de la diáfisis. b) Segunda falange del tercer digito: 1. Borde medial de la superficie articular proximal, 2. Borde máximo de la superficie articular proximal, 3. Borde lateral de la superficie articular proximal, 4. Ancho mínimo lateral de la diáfisis, 5. Borde del cóndilo lateral, 6. Borde mínimo de la superficie articular distal, 7. Borde del cóndilo medial de la superficie distal, 8. Ancho mínimo medial de la diáfisis.

en la zona de la diáfisis con las articulaciones distales y proximales anchas, la mayoría pertenecientes a la especie E. mexicanus (Figura 6a).

En el ACP de la 2FIII los dos primeros componentes explican el $75.48 \%$ de la varianza. El componente 1 reúne el $68.11 \%$ y el 2 el $7.37 \%$ de la varianza (Tabla 1). Con el primer componente se lograron separar dos grupos de forma clara, una forma rectangular, más estrecha en la articulación distal que en la articulación proximal con una mezcla de individuos de E. cedralensis y E. conversidens; y una forma cuadrada, con una anchura similar en las articulaciones distales y proximales donde la mayoría pertenecen a la especie E. mexicanus y con un individuo de E. conversidens y otro E. cedralensis; y aislada una forma cuadrada, con la articulación distal ligeramente más ancha que la proximal donde se encuentra la mayor parte de los individuos de E. conversidens mezclados con E. cedralensis y algunos E. mexicanus (Figura 6b).

En el AVC de MCIII encontramos diferencias significativas entre los grupos (Mahalanobis: $\mathrm{P}=0.0130$ ). Con el eje canónico 1 se pueden diferenciar dos grupos de forma, uno con los individuos de E. mexicanus y otro con los de E. cedralensis y E. conversidens, estas dos especies separadas por el eje canónico dos (Tabla 2, Figura 7a).

En el AVC de MTIII no encontramos diferencias significativas entre los grupos (Mahalanobis: $\mathrm{P}=0.0590$ ), posiblemente debido a que el grupo E. conversidens tiene un sólo representante. Sin embargo, con el eje canónico 1 se pueden diferenciar dos grupos de forma, uno con los individuos de E. mexicanus y otro con los especímenes de E. cedralensis; con el eje canónico 2 se puede diferenciar un único representante de E. conversidens (Tabla 2, Figura 7b).

En el AVC de 1FIII encontramos diferencias significativas entre los grupos (Mahalanobis: $\mathrm{P}=0.0001$ ). Con el eje canónico 1 se pueden diferenciar dos grupos de forma, uno con la mayor parte de los individuos de E. cedralensis y otro con los especímenes de E. mexicanus con algunos de E. cedralensis; con el eje canónico 2 se puede diferenciar un grupo con todos los individuos de E. conversidens (Tabla 2, Figura 8a).

En el AVC de 2FIII encontramos diferencias significativas entre los grupos (Mahalanobis: $\mathrm{P}=0.0001)$. Con el eje canónico 1 se pueden diferenciar dos grupos de forma, uno de la mayoría de los individuos de E. mexicanus y otro de E. conversidens; existe un tercer grupo con la mayor parte de los individuos de E. cedralensis y E. conversidens, separadas entre ellas por el eje canónico 2 (Tabla 2, Figura 8b).

\section{DISCUSIÓN}

Desde un punto de vista geológico, en LC-PT: la geomorfología es diversa con una presencia de numerosos edificios volcánicos y algunas

Tabla 1. Porcentaje de varianza individual y acumulado de los tres primeros componentes principales de los MCIII, MTIII, 1FIII y 2FIII. Abreviaturas: MCIII, metacarpo del tercer dígito; MTIII, metatarso del tercer dígito; 1FIII, primera falange del tercer dígito; $2 \mathrm{FIII}$, segunda falange del tercer dígito.

\begin{tabular}{lccc}
\hline $\begin{array}{l}\text { Elemento } \\
\text { postcraneal }\end{array}$ & $\begin{array}{c}\text { Componente } \\
\text { Principal }\end{array}$ & \% Varianza & Acumulada \% \\
\hline MCIII & 1 & 85.555 & 85.555 \\
& 2 & 5.211 & 90.766 \\
& 3 & 2.393 & 93.159 \\
MTIII & 1 & 56.886 & 56.886 \\
& 2 & 20.113 & 76.999 \\
& 3 & 9.889 & 86.888 \\
1FIII & 1 & 84.696 & 84.696 \\
& 2 & 4.487 & 89.182 \\
& 3 & 2.643 & 91.826 \\
2 FIII & 1 & 68.11 & 68.11 \\
& 2 & 7.373 & 75.484 \\
& 3 & 5.947 & 81.431 \\
\hline
\end{tabular}



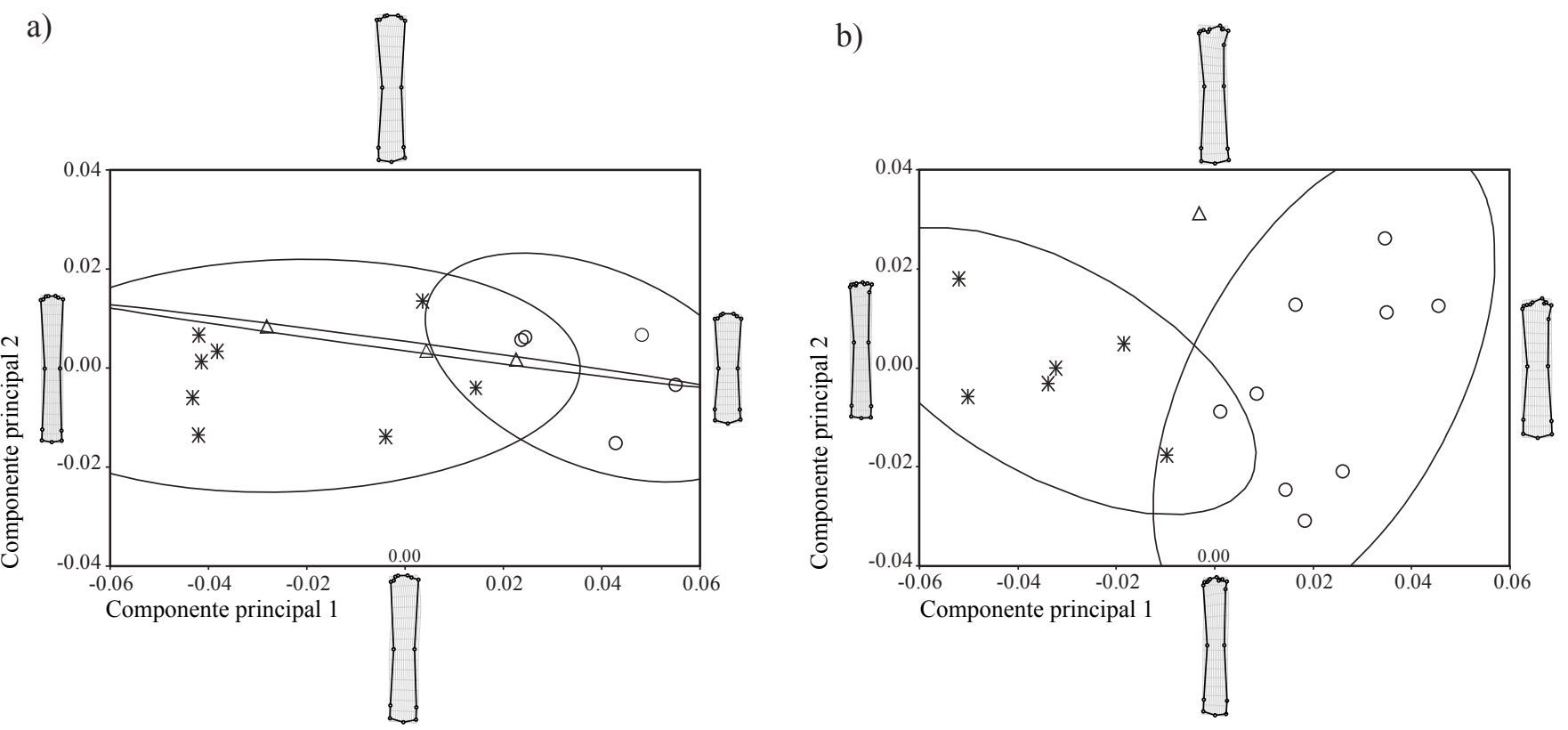

Figura 5. Representación gráfica del análisis de componentes principales, realizados sobre: a) MCIII, metacarpo del tercer dígito; b) MTIII, metatarso del tercer dígito. Elipses de densidad del 95\%. Símbolos: asterisco = Equus cedralensis; triángulo = E. conversidens; círculo = E. mexicanus.

zonas de planicies. La textura de los sedimentos (arcillas y limos) indica la presencia de un sistema léntico (Lago de Cuitzeo) (Marín-Leyva, 2011). Los restos de polen (Cheno-Am, Ambrosia spp., Asteraceae indet., Cirsium spp., and Thalictrum spp.) señalan la presencia de arbustos y herbáceas (Israde-Alcántara et al., 2010). En el caso de LPSA: la geomorfología es relativamente uniforme con grandes planicies y pocos edificios volcánicos. La textura de los sedimentos (arenas y limos) indica la presencia de un sistema lótico (río Lerma) (MarínLeyva, 2011). Actualmente no existen estudios de polen en el área. En el caso de CE: la geomorfología es homogénea dominada por una llanura plana rodeado de tres sierras. La textura de los sedimentos (arcillas y limos) así como la de los minerales indica la presencia de sistemas lénticos (lagunas y pantanos) y zonas aluviales (Flores-Díaz, 2012). Los restos de polen (Cupressus, Juniperus, Taxodium, Pinus, Quercus,
Poaceae y Asteraceae) señalan un cambio de ambiente frío y húmedo hacia templado y árido (Sánchez y Alvarado, 2012).

En el caso de la taxonomía, los análisis de MG realizados a los MCIII y MTIII comprueban que existen tres formas para esta estructura anatómica, una forma grácil y alargada (E. cedralensis), una robusta y alargada (E. mexicanus) y una forma intermedia (E. conversidens).

En el análisis de la 1FIII se comprueba también la existencia de tres grupos de forma: el grupo grácil (E. cedralensis), el robusto (E. mexicanus), bien diferenciados, y el mediano (E. conversidens). El grupo mediano parece tener características de forma similares a los otros dos grupos pero, al contar con un número mayor de muestras en comparación a MCIII y MTIII, se observa de manera más clara una separación de las otras dos formas. Sin embargo, se debe tener en a)

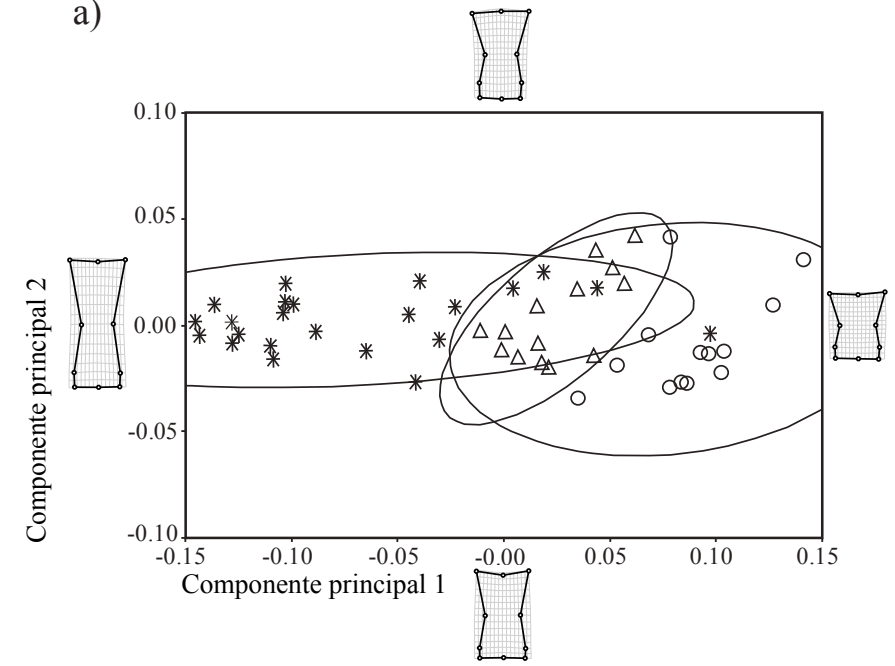

b)

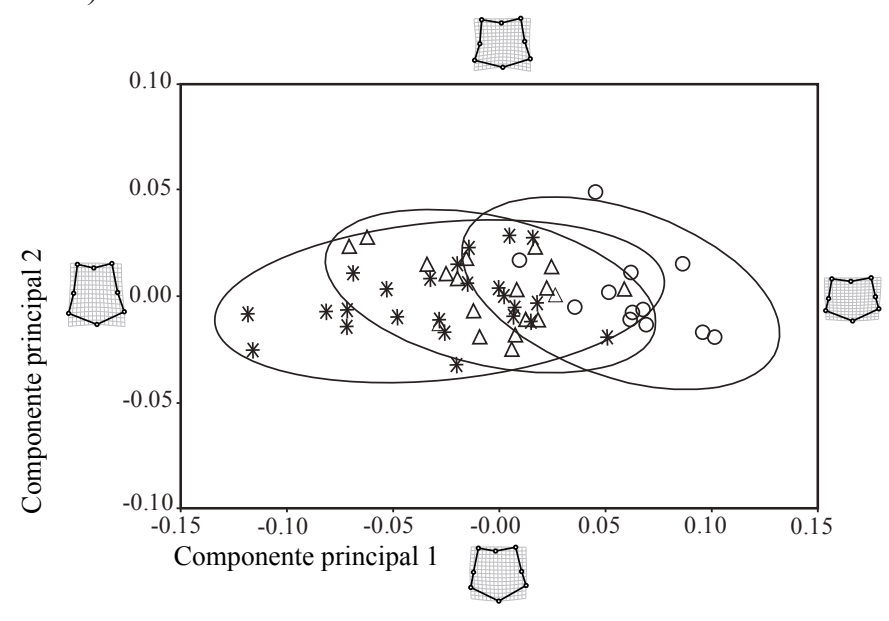

Figura 6. Representación gráfica del análisis de componentes principales, realizados sobre: a) 1FIII, primera falange del tercer dígito; b) 2FIII, segunda falange del tercer dígito. Elipses de densidad del 95\%. Símbolos como en la Figura 5. 
Tabla 2. Porcentaje de varianza individual y acumulado de los dos primeros ejes del análisis de varianza canónica de MCIII, MTIII, 1FIII y 2FIII. Abreviaturas: MCIII, metacarpo del tercer dígito; MTIII, metatarso del tercer dígito; 1FIII, primera falange del tercer dígito; 2FIII, segunda falange del tercer dígito.

\begin{tabular}{lccc}
\hline $\begin{array}{l}\text { Elemento } \\
\text { postcraneal }\end{array}$ & Variable canónica & \% Varianza & Acumulada \% \\
\hline MCIII & 1 & 86.955 & 86.955 \\
& 2 & 13.045 & 100 \\
MTIII & 1 & 89.180 & 89.180 \\
& 2 & 10.820 & 100 \\
1FIII & 1 & 63.575 & 63.575 \\
& 2 & 36.425 & 100 \\
2 FIII & 1 & 81.812 & 81.812 \\
& 2 & 18.188 & 100 \\
\hline
\end{tabular}

cuenta que la estructura de las 1FIII presenta diferencias, a veces muy acusadas, entre las falanges anteriores y las posteriores, las cuales son difíciles de separar cuando están aisladas, y por tanto al estudiarlas conjuntamente aumentan su variabilidad, dando lugar a grupos algo heterogéneos.

En el 2FIII se observa un solapamiento mayor entre los tres grupos, lo cual unido a las diferencias entre anteriores y posteriores (menores que en las 1FIII) da lugar a unos diagramas menos definitorios. Por todo ello, la 2FIII en muchos équidos, especialmente en el género Equus, no es un buen elemento óseo para utilizar en los análisis multivariantes (morfometría lineal o geométrica) (Alberdi et al., 2014).

De los análisis realizados sobre los MCIII, MTIII, 1FIII y 2FIII observamos que en los tres primeros se puede diferenciar bien la existencia de tres formas distintas, mientras que, en el cuarto, dichos grupos quedan altamente solapados. Esta mejor discriminación en MCTIII, MTIII y 1FIII en relación con la 2FIII se podría deber al mayor número de landmarks usados, lo que reflejaría mejor las formas analizadas, aunque posiblemente influyen más las pequeñas diferencias entre ellas.

Los resultados en relación con la ecomorfología y su vínculo entre forma y el sustrato muestran tres morfotipos relacionados con las tres especies (E. cedralensis, E. conversidens y E. mexicanus) cuyas características de forma en los elementos estudiados les pudieron dar la capacidad de habitar microambientes diferentes y, por lo tanto, poder vivir en el mismo macrohábitat.

Concretamente, el análisis de MG indica que la especie E. cedralensis tenía metápodos (MCIII y MTIII) alargados y gráciles. Las primeras (1FIII) y segundas (2FIII) falanges son ligeramente alargadas y angostas en la diáfisis, así como en la zona de la articulación distal, con lo que se podría deducir que las terceras falanges también fueran más estrechas (3FIII). Estas características morfológicas, también encontradas en las especies actuales (E. zebra y E. hemionus), Eisenmann (1984) las relaciona con ambientes abiertos o de montaña, en condiciones secas y suelos compactados, como podría ser un pastizal o un bosque abierto, paleo-hábitats que posiblemente existieron en las localidades en estudio (Alberdi et al., 2014; Marín-Leyva, 2011; Pérez-Crespo et al., 2009). Estos ambientes se corroboran con los análisis de polen que muestran la presencia de árboles, arbustos, herbáceas y pastos en LC-PT y CE (Israde-Alcántara et al., 2010; Sánchez y Alvarado, 2012). Además, los análisis de comportamiento alimenticio para esta especie en las tres localidades estudiadas indican una dieta de mediana a altamente abrasiva y con preferencia por el consumo de plantas $\mathrm{C}_{4}$, (Barrón-Ortiz et al., 2014; Marín-Leyva et al., 2016a, 2016b; Pérez-Crespo et al., 2009) lo que mostraría que para este équido la forma de las extremidades está relacionada con sus áreas preferidas de alimentación: zonas abiertas (pastizales).
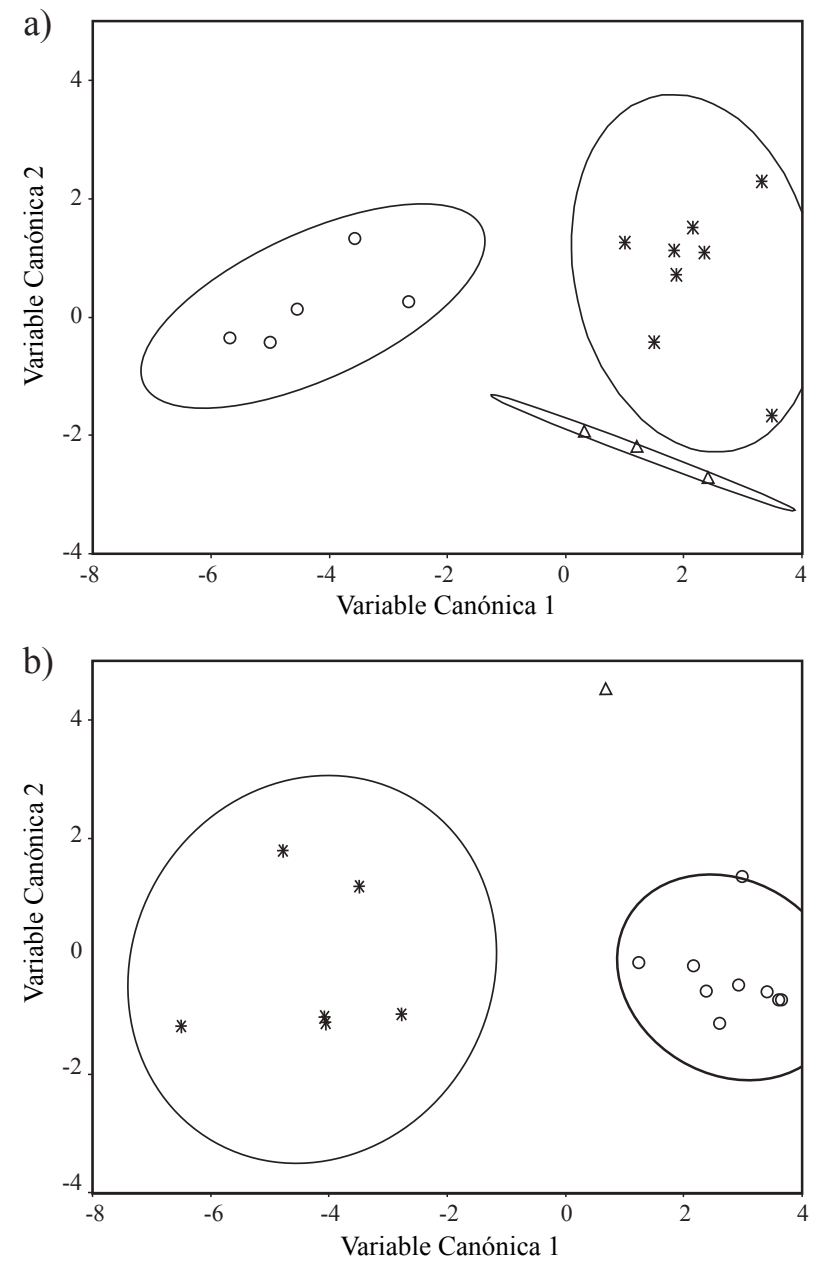

Figura 7. Representación gráfica del análisis de varianza canónica realizado sobre: a) MCIII, metacarpo del tercer dígito; b) MTIII, metatarso del tercer dígito. Elipses de densidad del 95\%. Símbolos como en la Figura 5.

Los análisis de MG muestran que la especie E. conversidens presentaba una forma intermedia en los huesos analizados en relación con E. cedralensis y E. mexicanus. Esta especie tenía metápodos (MCIII y MTIII) alargados, medianamente robustos y ligeramente anchos en las epífisis. Las primeras (1FIII) y las segundas (2FIII) falanges son medianamente alargadas y ligeramente robustas en la articulación distal por lo que se podría deducir que sus terceras falanges (3FIII) fueran algo más anchas que las de E. cedralensis. Estas características morfológicas, también encontradas en las especies actuales (E. hemionus, E. africanus y E. quagga), Eisenmann (1984) las relaciona con ambientes abiertos y cerrados, condiciones secas o húmedas y suelos compactados (no montañosos) o sueltos, como podría ser un pastizal, bosque abierto, bosque cerrado, paleo-hábitats que posiblemente existieron en las localidades en estudio (Alberdi et al., 2014; Marín-Leyva, 2011; PérezCrespo et al., 2009). En este caso, también está corroborado por los análisis de polen que estos ambientes muestran la presencia de árboles, arbustos, herbáceas y pastos en LC-PT y CE (Israde-Alcántara et al., 2010; Sánchez y Alvarado, 2012). Además, los análisis de dieta para esta especie en las localidades indican una dieta altamente abrasiva y con preferencia por el consumo de plantas $\mathrm{C}_{4}$ (Barrón-Ortiz et al., 2014; Marín-Leyva et al., 2016a, 2016b; Pérez-Crespo et al., 2009), lo que mostraría que a pesar de tener extremidades con las que podría habitar diferentes ambientes prefería las zonas abiertas: pastizales. 

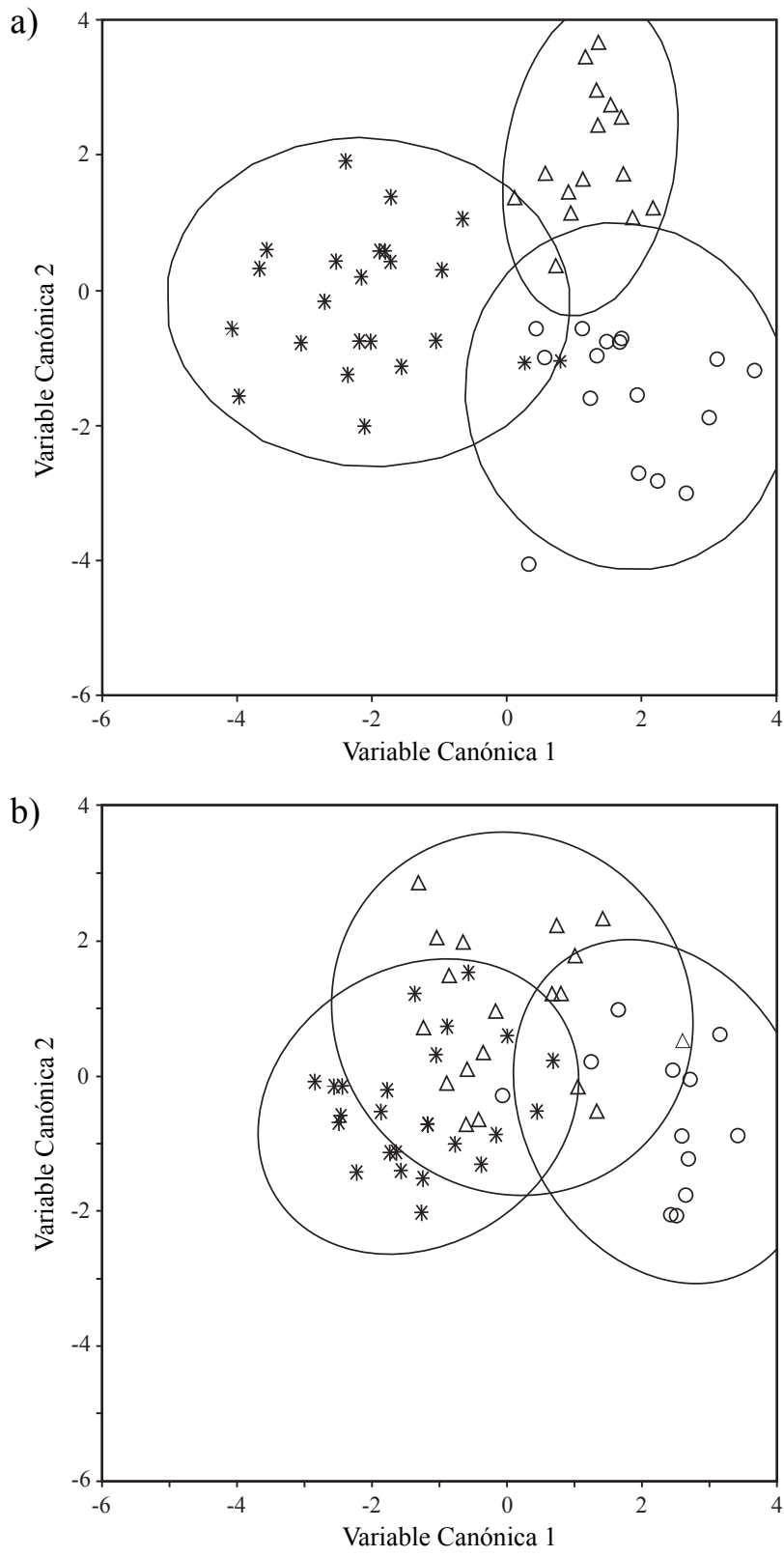

Figura 8. Representación gráfica del análisis de varianza canónica realizado sobre: a) 1FIII, primera falange del tercer dígito; b)2FIII, segunda falange del tercer dígito. Elipses de densidad del 95\%. Símbolos como en la Figura 5.

Los resultados del análisis de MG indican que la especie E. mexicanus tenía metápodos (MCIII y MTIII) alargados y muy robustos. Las primeras (1FIII) y segundas (2FIII) falanges son compactas, robustas y anchas en la articulación distal, lo que permitirían inferir que las terceras falanges fueran más anchas (3FIII). Para Eisenmann (1984) estas características morfológicas, también encontradas en las especies actuales (E. ferus y E. kiang), se relacionan con ambientes abiertos y cerrados, con condiciones húmedas y suelos poco compactados, como podrían ser pastizales, bosques abiertos, bosques, zonas de humedales e incluso con nieve; paleo-hábitats que posiblemente existieron en las localidades en estudio (Alberdi et al., 2014; Marín-Leyva, 2011; Pérez-Crespo et al., 2009). Los análisis de polen también muestran la presencia de árboles, arbustos, herbáceas y pastos en LC-PT y CE (Israde-Alcántara et al., 2010; Sánchez y Alvarado, 2012). Además, los análisis de dieta para esta especie en las localidades, indican una dieta de mediana a altamente abrasiva y con preferencia por el consumo de plantas $\mathrm{C}_{3}$ y $\mathrm{C}_{4}$ (Barrón-Ortiz et al., 2014; Marín-Leyva et al., 2016a, 2016b; Pérez-Crespo et al., 2009), lo que podría mostrar que para este équido la forma de las extremidades le daba la capacidad de moverse en diferentes ambientes abiertos (pastizales) y cerrados (bosques).

Sería muy interesante poder realizar el análisis de las terceras falanges mediante técnicas de MG ya que su forma parece estar muy ligada al substrato (Eisenmann, 1984). Desgraciadamente, los restos de los que disponemos aparecen alterados y/o fragmentados y son pocas las localidades donde se puede disponer de ejemplares completos. En este caso, de las tres localidades en estudio sólo disponemos de 3FIII completas en la localidad CE y no de las tres especies reseñadas.

Desde un punto de vista ambiental, los análisis de dieta meso y micro desgaste, así como los de isotopos estables de ${ }^{13} \mathrm{C}$ y ${ }^{18} \mathrm{O}$ de Mammuthus columbi, E. cedralensis, E. conversidens, E. mexicanus y Bison antiquus en la localidad LC-PT, muestran la existencia de plantas con componentes altamente abrasivos. Estas incluyen gramíneas o pastos $\left(\mathrm{C}_{4}\right)$ y elementos vegetales medianamente abrasivos como: árboles y arbustos $\left(\mathrm{C}_{3}\right)$, por lo que para este yacimiento existía un medio heterogéneo con ambientes cerrados (bosques) y abiertos (pastizales) (Díaz-Sibaja et al., 2018; Gutiérrez-Bedolla et al., 2016; Marín-Leyva et al., 2016a, 2016b). En el caso de LP-SA los análisis de dieta meso-micro desgaste así como los de isotopos estables de ${ }^{13} \mathrm{C} y{ }^{18} \mathrm{O}$ en Mammuthus columbi, E. cedralensis, E. conversidens, E. mexicanus, Bison antiquus en la localidad, muestran la existencia principalmente de plantas con componentes altamente abrasivos como las: gramíneas o pastos $\left(C_{4}\right)$ y en menor proporción elementos vegetales $C_{3}$, por lo que se deduce un ambiente heterogéneo pero con una mayor dominancia de ambientes abiertos (pastizales) con relación a los cerrados (bosques) (Díaz-Sibaja et al., 2018; Gutiérrez-Bedolla et al., 2016; Marín-Leyva et al., 2016a, 2016b). Mientras que en la localidad CE los análisis de dieta meso y micro desgaste, así como los de isótopos estables de ${ }^{13} \mathrm{C} y$ ${ }^{18} \mathrm{O}$ en Mammuthus columbi, Mammut americanum, E. cedralensis, E. conversidens, E. mexicanus, Tapirus sp., Bison sp., Glossotherium harlani, Glypthotherium sp. indican la existencia de plantas con componentes altamente abrasivos como las: gramíneas o pastos $\left(\mathrm{C}_{4}\right)$ y elementos vegetales medianamente abrasivos como: árboles y arbustos $\left(\mathrm{C}_{3}\right)$. Esto permite deducir para este yacimiento un ambiente heterogéneo con medios cerrados (bosques) y abiertos (pastizales) (Pérez-Crespo et al., 2009, 2012, 2014).

Las características paleoambientales encontradas en estudios posteriores con evidencia geológica, sedimentológica, palinológica y paleoecológica de especies asociadas en cada uno de los yacimientos, son corroboradas a partir de la información obtenida para los distintos morfotipos de caballos detectados en las localidades en estudio. De la comparación de las propiedades ambientales inferidas en este trabajo, relacionadas con los datos de Eisenmann (1984) para équidos actuales, se deduce que en las tres áreas en estudio dominarían ambientes heterogéneos, abiertos y cerrados, con suelos compactos y sueltos además de condiciones secas y húmedas.

\section{CONCLUSIONES}

El análisis de morfometría geométrica de los MCIII, MTIII y 1FIII corroboran la existencia de tres diferentes grupos de forma que se relacionan con las tres especies de caballos del Pleistoceno tardío en México. Se comprueba que la 2FIII no es un elemento significativo a la hora de llevar a cabo este tipo de estudios debido a la uniformidad de su forma o a la poca variabilidad de la misma.

El análisis de morfometría geométrica demuestra que existen 
caracteres diagnósticos, explicativos y poco explicativos para la diferenciación de las tres especies de caballos del Pleistoceno en México.

El MCIII se considera un carácter diagnóstico para diferenciar entre E. cedralensis y E. mexicanus, sin embargo, poco explicativo para discriminar E. conversidens (si bien disponemos de pocos restos). El MTIII se considera un carácter diagnóstico para diferenciar las tres especies, sin embargo, sólo se tiene un ejemplar de E. conversidens por lo que se deben hacer análisis con mayor número de muestras para comprobarlo. La 1FIII es un buen elemento para diferenciar entre E. cedralensis y E. mexicanus, y poco explicativo para discriminar $E$. conversidens. La 2FIII no es un carácter explicativo para diferenciar entre las tres especies.

Se observa la conveniencia de analizar las terceras falanges ya que serían un elemento muy interesante en este tipo de trabajos debido a que su forma parece estar muy ligada al substrato (Eisenmann, 1984). Sin embargo, en los sitios en estudio es un elemento que generalmente aparece alterado y/o fragmentado y son pocos los ejemplares completos, no suficientes para un estudio detallado.

Los resultados muestran tres morfotipos relacionados con tres especies (E. cedralensis, E. conversidens y E. mexicanus) y con la gracilidad de la parte distal de sus extremidades. Las características de sus respectivas formas les pudieron dar la capacidad de habitar microambientes diferentes y por lo tanto de poder vivir en el mismo macrohábitat. Además, debido a la variedad de morfotipos en las localidades en estudio se puede deducir que eran ambientes heterogéneos.

\section{AGRADECIMIENTOS}

Los autores agradecen a todos los estudiantes (Facultad de Biología, UMSNH) y los colegas de (Organización Especial de Investigación, La Piedad) que trabajaron en las campañas de campo en las localidades aquí reportadas. Igualmente al editor Alfredo Zurita y los dos árbitros anónimos, cuyas revisiones, comentarios y correcciones han ayudado a mejorar el manuscrito. Agradecemos también al Proyecto CGL201679334-P de la FICYT del MEICOMP de España. Y al laboratorio de Arqueozoología “M. en C. Ticul Álvarez Solórzano" INAH. Así como al Consejo Nacional de Ciencia y Tecnología (CONACYT) y al Consejo Técnico de la Investigación Científica, UNAM, por la beca posdoctoral DGAPA-PAPIIT para AHM-L.

\section{MATERIAL SUPLEMENTARIO}

En el suplemento electrónico se encuentran las tablas A1, A2, A3 y A4, pueden ser descargadas todas como archivo comprimido en el sitio web de la Revista <http://rmcg.unam.mx/>, dentro de la tabla de contenidos de este número.

\section{REFERENCIAS}

Alberdi, M.T., Ortiz-Jaureguizar, E., Prado, J.L., 1998, A quantitative review of european Stenonoid horse: Journal of Paleontology, 72(2), 371-387.

Alberdi, M.T., Arroyo-Cabrales, J., Polaco, O. J., 2003, ¿Cuántas especies de caballo hubo en una sola localidad del Pleistoceno Mexicano?: Revista Española de Paleontología, 18(2), 205-212.

Alberdi, M.T., Arroyo-Cabrales, J., Marín-Leyva, A.H., Polaco, O.J., 2014, Study of Cedral Horses and their place in the Mexican Quaternary: Revista Mexicana de Ciencias Geológicas, 31(2), 221-237.

Álvarez S.T., Polaco, O.J., 1982, Restos pleistocénicos de dos especies de Microtus (Rodentia: Muridae), del norte de San Luís Potosí, México: México, Anales de la Escuela Nacional de Ciencias Biológicas, 26, 47-53.
Barrón-Ortiz, C.R., Theodor, J.M., Arroyo-Cabrales, J., 2014, Dietary resource partitioning in the Late Pleistocene horses from Cedral, north-central Mexico: Evidence from the study of dental wear: Revista Mexicana de Ciencias Geológicas, 31(2), 260-269.

Bell, C.J., Lundelius Jr., E.L., Barnosky, A.D., Graham, R.W., Lindsay, E.H., Ruez Jr., D.R., Semken Jr., H.A., Webb, S.D., Zakrzewsky, R.J., 2004, Chapter 7: The Blancan, Irvingtonian and Rancholabrean Mammal Ages, en Woodburne, M.O., (ed.), Late Cretaceous and Cenozoic mammals of North America: Biostratigraphy and Geocronology: New York, EE.UU., Columbia University Press, 232-314.

Bignon, O., Baylac M., Vigne J-D., Eisenmann V., 2005, Geometric morphometrics and the population diversity of Late Glacial horses in Western Europe (Equus caballus arcelini): phylogeographic and anthropological implications: Journal of Archaeological Science, 32, 375-391.

Bookstein, F.L., 1991, Morphometric Tools for Landmark Data: Cambridge, Reino Unido, Cambridge University Press, 452 pp.

Bravo-Cuevas, V. M., Jiménez-Hidalgo, E., Priego-Vargas, J., 2011, Taxonomía y hábito alimentario de Equus conversidens (Perissodactyla, Equidae) del pleistoceno tardío (Rancholabreano) de Hidalgo, centro de México: Revista Mexicana de Ciencias Geológicas, 28(1), 65-82.

Caballero, M., Lozano-García, S., Vázquez-Selem, L., Ortega, B., 2010, Evidencias de cambio climático y ambiental en registros glaciales y en cuencas lacustres del centro de México durante el último máximo glacial: Boletín de la Sociedad Geológica Mexicana, 62(3), 359-377.

Calvo, M.D.M., Salesa, M.J., 2006, Estudio de la morfología geométrica dental del équido mioceno Anchitherium: implicaciones paleoecológicas: Estudios Geológicos, 62(1), 103-114.

Cervantes-Barriga, R., 2015, Especies del orden Carnivora del Rancholabreano de dos localidades del centro-occidente de México: Morelia, México, Universidad Michoacana de San Nicolás de Hidalgo, tesis de licenciatura, $82 \mathrm{pp}$.

Cervantes-Barriga, R., 2018, Paleodieta de Sigmodon hispidus (Rodentia, Cricetidae) en una localidad del Pleistoceno tardío del centro occidente de México: Morelia, México, Universidad Michoacana de San Nicolas de Hidalgo, tesis de maestría, $169 \mathrm{pp}$.

Christiansen, P., 2002, Locomotion in terrestrial mammals: the influence of body mass, limb length and bone proportions on speed: Zoological Journal of the Linnean Society, 136, 685-714.

Díaz-Sibaja, R., López-García, J.R., Arroyo-Cabrales, J., Jiménez-Hidalgo, E., Tejeda-Alvarado, F., Ponce-Saavedra, J., García-Zepeda, M.L., 2014, Cérvidos (Cervidae) del Rancholabreano de Michoacán-Guanajuato, en XII Congreso Nacional de Mastozoología, Puebla de los Ángeles, Puebla de Zaragoza, México: México, Asociación Mexicana de Mastozoología, A.C., Memorias congreso (paleontología), Resumen, 1.

Díaz-Sibaja, R., Jiménez-Hidalgo, E., Ponce-Saavedra, J., García-Zepeda, M. L., 2018, A combined mesowear analysis of Mexican Bison antiquus shows a generalist diet with geographical variation: Journal of Paleontology, 92(6), 1130-1139.

Eisenmann, V., 1984, Sur quelques caracteres adaptatifs du squelette d'Equus (Mammalia, Pesissodactyla) et leurs implications paleoecologiques: Bulletin du Muséum National d'Histoire Naturelle, Paris, $4^{\circ}$ ser., 6 , section C, $n^{\circ} 2,185-195$.

Eisenmann, V., Alberdi M. T., de Giuli C., Staesche., 1988, Collected papers after the "New York International Hipparion Conference, 1981", en Woodburne, M., Sondaar, P., (eds.) Studyng Fossil Horses, I, Methodology: Leiden, Netherlands, E.J. Brill Leiden, 72 pp.

Eng-Ponce, J., 2018, Perezosos (Xenarthra: Folivora) y capibaras (Rodentia: Hydrochoerinae) del yacimiento pleistocénico (Rancholabreano) de la Cinta-Portalitos, Michoacán-Guanajuato, México: Morelia, México, Universidad Michoacana de San Nicolas de Hidalgo, tesis licenciatura, $120 \mathrm{pp}$.

Ferrusquía-Villafranca, I., Arroyo-Cabrales, J., Martínez-Hernández, E., Gama-Castro, J., Ruiz-González, J., Polaco, O.J., Johnson, E., 2010, Pleistocene mammals of México: A critical review of regional chronofaunas climate change response and biogeographic provinciality: Quarterary International, 212, 53-104.

Fink, W., 1990, Data acquisition in systematic biology en Rohlf, F.J., Bookstein, F.L., (eds.) Proceedings of the Michigan Morphometrics Workshop held 
at the University of Michigan: Ann Arbor, EE.UU., The University of Michigan Museum of Zoology, 9-21.

Flores-Díaz, A., 2012, Cambios paleoclimaticos durante el PleistocenoHoloceno en un área semidesértica, Cedral, en Mirambell, L.E. (ed.), Rancho "La Amapola" Cedral, un sitio arqueológico-paleontológico pleistocénico-holocénico con restos de actividad humana: Ciudad de México, México, Instituto Nacional de Antropología e Historia, 87-146.

García-Zepeda, M.L., 2006, Nuovi dati Paleontologici dalla depressione lacustre di Cuitzeo Michoacán, México: Firenze, Toscana, Italia, Universita degli studi di Firenze, tesis doctoral, $115 \mathrm{pp}$.

Garland, T., Janis, C.M., 1993, Does metatarsal/femur ratio predict maximal running speed in cursorial mammals?: Journal of Zoology 229, 133-151.

Gregory, W.K., 1912, Notes on the principles of quadrupedal locomotion and on the mechanism of the limbs in hoofed animals: Annals of the New York Academy of Sciences 22, 267-294.

Gutiérrez-Bedolla, M., García-Zepeda, M.L., López-García, R., ArroyoCabrales, J., Marín-Leyva, A.H., Meléndez-Herrera, E., Fuentes-Farías, A.L., 2016, Diet and habitat of Mammuthus columbi (Falconer, 1857) from two Late Pleistocene localities in central western Mexico: Quaternary International, 406, 137-146.

Hammer, O, Harper, D.A.T., 2006, Paleontological Data Analysis: Malden, EE.UU., Blackwell Publishing Ltd, 351 pp.

Heintzman, P.D., Zazula, G.D., MacPhee, R.D.E., Scott, E., Cahill, J.A., McHorse, B.K., Kapp, J.D., Stiller, M., Woller, M.J., Orlando, L., Southon, J., Froese, D.G., Shapiro, B., 2017, A new genus of horse from Pleistocene North America: eLife, 6, 1-43.

Hibbard, C.J., 1955, Pleistocene vertebrates from the Upper Becerra (Becerra Superior) Formation, valley of Tequixquiac, Mexico, with notes on the other Pleistocene forms: University of Michigan, Museum of Paleontology Contributions, 12, 47-96.

Israde-Alcántara, I., Garduño-Monroy, V.H., Ortega-Murillo, R., 2002, Paleoambiente lacustre del Cuaternario tardío en el centro del lago de Cuitzeo: Hidrobiologica, 2, 61-78.

Israde-Alcántara, I., Velázquez-Durán, R., Socorro, M., García, L., Vázquez, G. D., Garduño-Monroy, V.H., 2010, Evolución Paleolimnológica del Lago Cuitzeo, Michoacán durante el Pleistoceno-Holoceno: Boletín de la Sociedad Geológica Mexicana, 62(3), 345-357.

MacFadden, B.J., 1992, Fossil Horses, Systematics, Paleobiology, and Evolution of the Family Equidae: Cambridge, Reino Unido, Cambridge University Press, 369 pp.

Marín-Leyva A.H., 2011, Caballos del Pleistoceno y sus paleoambientes en dos cuencas de Michoacán: Morelia, Michoacán, México: Morelia, México, Universidad Michoacana de San Nicolás de Hidalgo, Facultad de Biología, tesis maestría, $167 \mathrm{pp}$.

Marín-Leyva, A.H., Arroyo-cabrales, J., García-Zepeda, M.L., Ponce-Saavedra, J., Schaaf, P., Pérez-Crespo, V.A., Morales-Puente, P., Cienfuegos-Alvarado, E., Alberdi, M.T., 2016a, Feeding ecology and habitat of Late Pleistocene Equus horses from west-central Mexico using carbon and oxygen isotopes variation. Revista Mexicana de Ciencias Geológicas, 33(2), 157-169.

Marín-Leyva, A.H., DeMiguel, D., García-Zepeda, M.L., Ponce-Saavedra, J., Arroyo-Cabrales, J., Schaaf, P., Alberdi, M.T., 2016b, Dietary adaptability of Late Pleistocene Equus from West Central Mexico: Palaeogeography, Palaeoclimatology, Palaeoecology, 441, 748-757.

Mirambell, E.L., 2012, Las excavaciones en Rancho "La Amapola" Cedral, un sitio arqueológico-paleontológico, pleistocénico- holocénico con restos de actividad humana, en Mirambell, L.E. (ed.), Rancho "La Amapola" Cedral, un sitio arqueológico-paleontológico pleistocénico-holocénico con restos de actividad humana: Ciudad de México, México, Instituto Nacional de Antropología e Historia, 31-70.

Moreno Flores, J.O., 2018, Herpetofauna del Pleistoceno tardío de La CintaPortalitos, Michoacán-Guanajuato, Mexico: Morelia, México, Universidad Michoacana de San Nicolás de Hidalgo, tesis de licenciatura, 120 pp.

Olivera-Carrasco M.T., 2012, Moluscos continentales de Cedral, un sitio del Pleistoceno final de México en Mirambell, L.E. (ed.), Rancho "La Amapola" Cedral, un sitio arqueológico-paleontológico pleistocénico-holocénico con restos de actividad humana: Ciudad de México, México, Instituto Nacional de Antropología e Historia, 225-284.

Owen, R., 1869, On fossil remains of Equines from Central and South America referable to Equus conversidens Ow. E. tau Ow., and E. arcidens Ow:
Philosophical Transactions of Royals Society of London, 159, 559-573.

Pérez-Crespo, V.A., Sánchez C.B., Arroyo-Cabrales, J., Alberdi, M.T., Polaco, O.J., Santos-Moreno, A., Morales-Puente, P., Benammi, M., CienfuegosAlvarado, E., 2009, La dieta y el hábitat del mamut y los caballos del Pleistoceno tardío de El Cedral: Revista Mexicana de Ciencias Geológicas, 26(2), 347-355

Pérez-Crespo, V.A., Arroyo-Cabrales, J., Alva-Valdivia, L.M., Morales-Puente, P., Cienfuegos-Alvarado, E., 2012, Diet and habitat definitions for Mexican glyptodonts from Cedral (San Luis Potosí, México) based on stable isotope analysis: Geological Magazine, 149(1), 153-157.

Pérez-Crespo, V.A., Alva-Valdivia, L.M., Arroyo-Cabrales, J., Morales-Puente, P., Cienfuegos-Alvarado, E., Otero, F. J., 2014, Marcadores Biogeoquímicos de $\delta^{13} \mathrm{Cy} \delta^{18} \mathrm{O}$ : Inferencia sobre dieta y hábitat de mamíferos que habitaron en el Pleistoceno tardío en México: Ciudad de México, México, Monografías del Instituto de Geofísica, Universidad Nacional Autónoma de México, $53 \mathrm{pp}$.

Pérez-Gonzáles, M.S., Godínez-García, V., 2007, Pequeños Vertebrados Fósiles y La Bioestratigrafía de La Cinta, Michoacán y Portalitos, Guanajuato: Morelia, México, Universidad Michoacana de San Nicolás de Hidalgo, Facultad de Biología, tesis de licenciatura, $93 \mathrm{pp}$.

Plata-Ramírez, R.A., 2012, Camellos Fósiles de La Cinta-Portalitos y La Piedad-Santa Ana Michoacán y Guanajuato, México: Morelia, México, Universidad Michoacana de San Nicolás de Hidalgo, Facultad de Biología, tesis licenciatura, $70 \mathrm{pp}$.

Prado L.J., Alberdi M.T., 1994, A Quantitative Review of the Horses Equus from South America: The Palaentological Association, Palaeontology, 37(2), 459-481.

Priego-Vargas, J., Bravo-Cuevas, V.M., Jiménez-Hidalgo, E., 2016, The record of Cenozoic horses in Mexico: current knowledge and palaeobiological implications: Palaeobiodiversity and Palaeoenvironments, 96(2), 305-331.

Reyes, C.M., Flores, D.A., 2012, La geología del área de Cedral en Rancho "La Amapola" Cedral, un sitio arqueológico-paleontologico, pleistocenicoholocenico con restos de actividad humana, en Mirambell, L.E. (ed.), Rancho "La Amapola" Cedral, un sitio arqueológico-paleontológico pleistocénico-holocénico con restos de actividad humana: Ciudad de México, México, Instituto Nacional de Antropología e Historia, 21-30.

Rubenstein, D.I., 2011, Family Equidae (Horses and Relatives), en Wilson D.E., Mittermeier, R.A., (eds.), Handbook of the Mammals of the World vol. 2, Hoofed Mammals: Barcelona, España, Lynx Editions, 139-143.

Sánchez M.F., Alvarado J.L., 2012, Análisis palinológico en Rancho "La Amapola" Cedral, un sitio arqueológico-paleontológico, pleistocénicoholocénico con restos de actividad humana, en Mirambell, L.E. (ed.), Rancho "La Amapola" Cedral, un sitio arqueológico-paleontológico pleistocénico-holocénico con restos de actividad humana: Ciudad de México, México, Instituto Nacional de Antropología e Historia, 285-294.

Slice D.E., 2007, Geometric Morphometrics: Annual Review of Anthropology, $36,261-81$.

Steudel, K., Beattie, J., 1993, Scaling of cursoriality in mammals: Journal of Morphology 217, 55-63.

Van Asperen E.N., 2010, Ecomorphological adaptations to climate and substrate in late Middle Pleistocene caballoid horses: Palaeogeography, Palaeoclimatology, Palaeoecology 297, 584-596.

Van Valkenburgh, B., 1987, Skeletal indicators of locomotor behaviour in living and extinct carnivores: Journal of Vertebrate Paleontology 7, 162-182.

Winans, M.C., 1985, Revision of North American fossil species of the genus Equus (Mammalia: Perissodactyla: Equidae): Austin, EE.UU., University of Texas, tesis doctoral, $264 \mathrm{pp}$.

Winans, M.C., 1989, A quantitative study of the North America fossil species of the genus Equus, en Prothero, D.R., Schoch R.M., (eds.), The Evolution of the Perissodactctyls: Oxford, Reino Unido, Oxford University Press, 263-297.

Zelditch, M.L., Swiderski, D.L., David Sheets, D.H., Fink W.L., 2004, Geometric Morphometrics for Biologists: a Primer: San Diego, EE.UU., Elsevier Academic Press, 456 pp.

Manuscrito recibido: septiembre 13, 2018

Manuscrito corregido recibido: febrero 20, 2019

Manuscrito aceptado: febrero 25, 2019 